\title{
Propuesta de una evaluación integral para el Bachillerato de la enseñanza del inglés en la Escuela de Literatura de la Universidad Nacional de Costa Rica
}

\author{
Natin Gerardo Guzmán Arce, Universidad Nacional de Costa Rica, Costa Rica \\ Mayra Loaiza Berrocal, Universidad Nacional de Costa Rica, Costa Rica
}

\begin{abstract}
Resumen: La propuesta que se presenta en este artículo tiene como objetivo principal establecer una coordinación evaluativa de las materias de los diferentes cursos impartidos en el Bachillerato de la Enseñanza del Inglés (BEI) de la Escuela de Literatura y Ciencias del Lenguaje (ELCL) de la Universidad Nacional de Costa Rica (UNA). Esta propuesta de Evaluación Integral tiene el fin de lograr un vínculo entre los instructores, objetivos de los cursos y el aprendizaje del idioma per se. La evaluación integral busca que los aprendices del idioma inglés puedan ver la correlación del idioma de una manera cíclica y no aislada. En otras palabras, que el estudio de la gramática, fonética, cultura, la expresión y producción oral y escrita estén vinculadas entre sí a través de trabajos en común (proyectos) que contribuyan a la formación académica y que contribuyan al aprendizaje. Esto requiere de un estudio profundo de los planes de estudios, de los temas y objetivos y de la comunicación permanente de los académicos. La idea es establecer dicha propuesta como un eje transversal de trabajo permanente, y que se establezca como una actividad de enseñanza para los alumnos en cada ciclo lectivo en su respectivo nivel.
\end{abstract}

Palabras clave: comunicación, estudios lingüisticos, educación, adquisición de una segunda lengua, currículum, métodos de enseñanza y evaluación

\begin{abstract}
It is the main objective of this proposal to carry out a coordination of an Integral Evaluation within all the subjects taught in the Teaching English Major provided by the Faculty of Literature, Language and Science in the National University of Costa Rica. This proposal aims to involve all parties: faculty members, professors and students in order to obtain much better results in the learning process of the English Language. This Integral Evaluation procedure intends to help students see the learning of the language as a whole contemplating oral, written, spoken and listening and comprehension production as a unique element as well as a result throughout the different tasks (projects) given to students based on the topics and course objectives. The main goal is to establish this proposal as a regular activity in the major depending on the different major levels.
\end{abstract}

Keywords: Communication, Linguistics studies, Second Language Acquisition, Syllabus, Teaching Methods and Evaluation

\section{Introducción}

$\mathrm{L}$ a Universidad Nacional de Costa Rica es una de las cuatro universidades públicas que emergieron por la necesidad de tener un pueblo altamente educado y letrado. A la par de la Universidad de Costa Rica, el Instituto Tecnológico de Costa Rica y la Universidad Técnica Nacional; La Universidad Nacional de Costa Rica (UNA) ha tratado de extender su rama de estudios a todos los sectores del pueblo costarricense, especialmente a poblaciones de zonas rurales y de un nivel socio económico bajo.

En sus inicios, la UNA fue una casa de estudios llamada Escuela Normal de Costa Rica cuyo objetivo primordial era formar bachilleres en educación (maestros y maestras) quienes llevarían el saber a todo el territorio nacional por la necesidad de satisfacer la escasez de profesores y profesoras para la enseñanza media del país. La UNA pasa de ser la Escuela Normal de Costa Rica a formar una institución educativa de grado universitario el 15 de febrero de 1973. Su creación estuvo envuelta en debates y oposición por algunos intelectuales y grupos políticos, pero la batalla da sus

Revista Internacional de Ciencias Humanas

Volumen 5, Número 2, <http://lascienciashumanas.com>, ISSN 2530-4526

(C) Global Knowledge Academics. Natin Gerardo Guzmán Arce, Mayra Loaiza Berrocal.

Todos los derechos reservados. Permisos: soporte@gkacademics.com

Republicado de Revista Internacional de Humanidades en la Educación 5(2), 2016 (pp. 1-12) 
frutos y surge una nueva Alma Máter para que los costarricenses de entonces y de ahora opten por una educación superior estatal.

La UNA se ha abocado por una educación humanista donde su lema: "La verdad nos hace libres", es la muestra misma de una educación abierta, social y de respeto solidario al ser. Aunque al principio, su primordial objetivo era la formación de docentes para primaria y secundaria; el crecimiento socio-político costarricense abre las posibilidades de expansión educativa y formación profesional. En la actualidad, la Universidad Nacional de Costa Rica cuenta con carreras educativas en lo social, técnico, educativo, deportivo, investigativo entre otras. Se ha expandido por todo el país y tiene un total de siete sedes universitarias. Es una de las universidades más importantes de Latinoamérica (ocupa lugar 66) y goza de gran prestigio internacional (en el ranquin-lugar 1318 a nivel mundial). A su haber, están miles de costarricenses quienes han dejado sus aulas y han hecho de su conocimiento la Costa Rica del siglo XXI.

En el afán de siempre seguir mejorando, la UNA ha venido revalorando los programas de estudios de las carreras, y en la actualidad cuenta con catorce de sus carreras acreditas internacionalmente, dentro de la cuales se encuentra el Bachillerato de la Enseñanza del Inglés (BEI). Este bachillerato es una de las carreras de mayor demanda de la universidad. Su malla curricular le permite al discente desarrollar sus destrezas en el idioma inglés desde un nivel cero hasta poder alcanzar el dominio de la segunda lengua. El perfil de salida de este bachillerato tiene como premisa formar docentes quienes puedan enseñar el idioma en los diferentes centros educativos de secundaria del país a nivel público, privado o semiprivado.

A pesar de los buenos resultados en el tipo de profesional graduado de BEI, siempre se busca mejorar el sistema evaluativo y pedagógico de la carrera como parte de plan de mejoramiento correspondiente a la acreditación. Durante el primer año de la carrera, los estudiantes obtienen cursos integrados donde ven el aprendizaje del idioma de forma holística (evaluación integral). Sin embargo, a partir del segundo año, la enseñanza del idioma se divide por especialidad donde los estudiantes reciben cursos por separados tales como: gramática, redacción y escritura, producción oral y auditiva, cursos de lectura, cultura, lingüística y todo aquello que implica el aprendizaje de un idioma.

La distribución anteriormente mencionada, no presenta ningún problema; ya que, todo plan de estudios tiene una estructura parecida. No obstante, una evaluación integral por medio de proyecto podría mejorar significativamente la adquisición del inglés como segundo idioma de una forma más precisa, asertiva y con resultados lingüísticos aún más positivos. Por tanto, el objetivo primordial de este artículo es esbozar una propuesta de evaluación y educación integral en los cuatro niveles del plan de estudios del BEI que se planteará al área de académicos de la Escuela de Literatura y Ciencias del Lenguaje (ELCL) de la Universidad Nacional de Costa Rica. Dicha propuesta nace como el resultado de las discusiones y argumentos llevados a cabo en las reuniones de los académicos de la carrera del BEI.

\section{Justificación de la propuesta}

Las propuestas educativas de la Escuela de Literatura y Ciencias del Lenguaje siempre están en constante escrutinio por los académicos quienes tienen la obligación de impartir el conocimiento y evaluar a los discentes. Durante los últimos años, los doctos de la ELCL han estado discutiendo la necesidad de integrar los cursos de los diferentes niveles de la carrera, sin embargo, tomar decisiones al respecto se torna un poco complicado ante la carencia de una propuesta pasible. Ponerse de acuerdo requiere de mucha constancia, colaboración, comunicación y equipo de trabajo. No se debe confundir "trabajar en equipo" con "equipo de trabajo". Son dos conceptos que van de la mano, pero que poseen diferentes significados. El equipo de trabajo implica el grupo humano en sí, cuyas habilidades y destrezas permitirán alcanzar el objetivo final. Sus integrantes deben estar bien organizados, tener una mentalidad abierta y dinámica alineada con la misión y visión que les envuelve. Esta propuesta tiene como iniciativa una integración más global donde los estudiantes vean una coherencia intrínseca en el desarrollo de los programas de cada curso. Los estudiantes del BEI han demostrado una y otra vez, que cuando usan la lengua meta tienden a separar lo aprendido en un curso de otro. Por ejemplo, muchas veces lo estudiado en el curso de gramática, no necesariamente se refuer- 
za en las clases de los cursos orales o de composición, donde por lo contrario, dichas materias deberían integrar todos los conocimientos de una manera eficaz. La oportunidad de llevar a cabo una evaluación integral por medio de un proyecto, podría ser la oportunidad perfecta para que los estudiantes se vean obligados a integrar la sapiencia obtenida. Además, dicho proyecto ayudará a mejorar su nivel de lengua; ya que al integrar todos los conocimientos podrán internalizar el idioma de una forma holística. Por tanto, la puesta en común de una evaluación integral por medio de un proyecto tendría beneficios invaluables para el aprendizaje en la enseñanza de un segundo idioma. Sin embargo, ¿qué es una evaluación integral? ¿Cómo funciona? ¿Cuáles son los puntos a tomar en cuenta para llevarla a cabo? Estas interrogantes serán examinadas en el siguiente apartado.

\section{Evaluación integral}

En el vasto concepto del término, la evaluación integral es el resultado de uso de múltiples metodologías de enseñanza en los diferentes sistemas educativos mundiales con éxito o no que han dejado una pequeña o gran huella en las diferentes áreas del sistema mismo. El bilingüismo o bien el poliglotismo nunca han tenido un auge tan relevante (debido a la globalización) como en los primeros años del siglo XXI. Los países han hecho grandes esfuerzos económicos y sociales para crear sociedades bilingües especialmente inglés y el idioma nativo de cada sociedad.

Son vastas las teorías expuestas de cómo se aprende o debe enseñarse un segundo idioma. Dentro de las más relevantes se encuentra la teoría de Stephen D. Krashen que se basa en cinco hipótesis: la hipótesis de la adquisición-aprendizaje, del monitor, del orden natural, de entrada y por último, la hipótesis filtro afectivo (1985). Siendo Krashen un pionero en el estudio y análisis del aprendizaje de un segundo idioma, él mismo y muchos de sus seguidores presentaron diversos métodos de enseñanza tradicionales como la instrucción gramatical o magistral hasta el aprendizaje tipificado por las inteligencias múltiples expuesto por Howard Gardner (1988). Contribuciones como estás han logrado esbozar un sinfín de metodologías que coadyuvan entre sí para la enseñanza $\mathrm{y}$ amaestramiento de un segundo idioma.

La enseñanza de un segundo idioma al igual que cualquier otra disciplina de la educación ha evolucionado y la tendencia gira en un sistema educativo integral. En palabras de Ludwig Wettgenstein "Los límites de mi lenguaje significan los límites de mi mundo" (Tractatus LogicoPhilosophicus, 1999, \& 5.6). Esta frase envuelve las nuevas metodologías y el fin principal de la educación, el hacer del educando un ser integral y funcional para la sociedad donde vive y el mundo. Tal perspectiva sirve como plataforma para que los sistemas educativos busquen una mayor integridad en todas las competencias de la instrucción formal. Por tanto, aprender un segundo idioma no debe limitarse a sólo poder comunicase y expresarse; sino ser lingüísticamente, socialmente y culturalmente preciso y asertivo.

Esta orientación educativa fortalece la evaluación integral en la enseñanza de un segundo idioma en cualquier programa de estudios. Por evaluación integral se entiende lo expuesto por Leanna Triall (1995) quien lo conceptualiza en dos dimensiones: la primera es valoración (assessment) que es el proceso de observación y acumulación objetiva de evidencias de progreso individual de los estudiantes, y otra la evaluación; proceso en el cual se hacen estimaciones acerca de la pertinencia y efectividad de la enseñanza para el aprendizaje. En la definición de Triall se presentan los dos ejes fundamentales de la evaluación integral donde el aprendiz está en constante auto valoración de su conocimiento y proceso.

En la teoría una evaluación integral es clara, sin embargo, en la práctica su funcionabilidad se puede tornar algo compleja. Evaluar integralmente requiere del acuerdo de varios elementos: el programa de estudios, coordinación de los académicos y la guía dirigida de los estudiantes. Todo programa de estudios tiene como objetivo formar profesionales de calidad en el área de estudio. En el BEI de la Escuela de Literatura y Ciencias del Lenguaje de la UNA, dicho objetivo esta intrínsecamente ligado en todos los niveles. Los cursos están dirigidos al aprendizaje del idioma así como a preparar a los aprendices en la formación educativa. Una evaluación integral fortalecería ambas, además que complementaría al aprendiz como ser profesional. ¿Cómo llevar a cabo esta evaluación? 
Es aquí donde entra en juego la coordinación de los elementos. Por lo tanto, la propuesta sugiere implantar una evaluación por medio de proyectos establecidos a nivel horizontal en el nivel dado y basados en los tópicos estudiados en los cursos. De acuerdo con el Ministerio Nacional de Educación de Colombia (1999), dentro de las características y principios de la evaluación integral por medio de proyectos o tareas están:

1- Se basa en la libertad y democracia de la selección de temas.

2- La metodología se centra en el estudiante y su capacidad y desarrollo.

3- La investigación es el procedimiento más usado para desarrollar los temas y proyectos.

4- Se da gran importancia a la contextualización, significado y propósito de los proyectos de manera que contribuyan al desarrollo de un todo coherente.

5- La cooperación entre los estudiantes, representada en el trabajo grupal, es de gran importancia ya que permite la socialización, la negociación, el consenso, el respeto por las diferencias y el logro de un trabajo armónico con un producto común.

6- La autonomía y responsabilidad de los estudiantes en la planeación y ejecución de tareas permite el desarrollo de comportamientos y actitudes positivas y de gran valor para su vida futura.

7- Se da igual valoración a los procesos y a sus productos, en la creencia de que un proceso entusiasta, serio, responsable y bien llevado debe tener como resultado un producto de características similares y de igual valor.

8- El carácter interdisciplinario de la metodología es uno de sus rasgos más importantes; los temas se exploran desde la perspectiva de diferentes áreas, permitiendo a los estudiantes tener una visión total de ellos, más acorde con la realidad. Esta manera de desarrollar los temas requiere también una integración de los docentes para planear sus clases.

9- Todo lo anterior contribuye a la formación integral del aprendiz, ya que a la vez que desarrolla sus habilidades lingüísticas refuerza la adquisición y el desarrollo de conceptos y de habilidades cognoscitivas y sociales y la formación de una competencia que permita establecer, coordinar y cultivar relaciones con los demás.

Definitivamente estas características y principios precisan el punto de partida para que los elementos y participantes (académicos y estudiantes) colaboren entre sí para llevar a cabo la evaluación integral. Basado en los beneficios que se obtienen de una evaluación integral por medio de proyectos o tareas, es que se propone implementarla en los niveles del BEI de la ELCL de la Universidad Nacional de Costa Rica. Dicho planteamiento se formula como una proposición de trabajo que se puede instaurar paulatinamente y primero como plan piloto donde su ejecución esté sujeta a cambios y mejoramientos en el procedimiento de implementación.

\section{La propuesta educativa}

\section{Evaluación integral por medio de proyectos o tareas}

La evaluación integral por medio de proyectos o actividades se ha vuelto muy común especialmente en los cursos de la enseñanza de idiomas. En sus inicios más primitivos es aprender haciendo. $\mathrm{Su}$ desarrollo ha elaborado métodos y técnicas más complejos como: aprendizaje por experimentos o tareas (task-based or content-based approaches). El aprender haciendo (del inglés learnng by doing) cobra especial importancia dentro de esta metodología. Igualmente importante es la relación estrecha que se establece entre la clase de lengua y las demás áreas, hasta el punto en que el aprendizaje y la enseñanza no se presentan necesariamente en lecciones individuales sino que se integran en temas que se desarrollan de manera activa, generalmente a través de trabajos o procedimientos llevados a cabo por los discentes.

Teniendo en cuenta las características y los principios de lo que es evaluación integral y las metodologías nacidas en el proceso de enseñanza-aprendizaje de la misma en los programas de estudios del inglés como segundo idioma, la propuesta que se plantea en este artículo para llevar a cabo una evaluación integral en el BEI en la ELCL de la UNA, se expone a continuación en detalle. 
Primero es importante señalar que el Bachillerato en la Enseñanza del Inglés (BEI) está diseñado para que los estudiantes (quienes en su mayoría tienen un nivel de inglés bajo) logren aprender el idioma así como recibir la pedagogía necesaria para llegar a ser profesionales de la educación en el área de inglés a nivel nacional. La malla curricular del BEI está divida por niveles, contempla los cursos de lengua pura y los pedagógicos, (estos últimos compartidos con la Escuela de Educación). La carrera en sí tiene una duración de cuatros años para que un estudiante obtenga el grado de bachiller.

Durante el primer año (I Nivel) de la carrera del BEI, los estudiantes deben llevar dos cursos denominados Inglés Integrado I y II. Cada curso tiene una duración de 17 semanas y el año se divide en dos ciclos según lo establece el estatuto orgánico de la UNA. Sendos cursos integran la parte pedagógica y las áreas del idioma; sin embargo, segregan la parte evaluativa por áreas. Los cursos de Inglés Integrado I y II son impartidos por dos docentes donde uno se encarga de enseñar y evaluar las habilidades de escucha, habla y lectura, mientras que el otro docente se encarga de enseñar y evaluar las habilidades de la gramática y la producción escrita. Actualmente, la evaluación es a discreción de cada docente siguiendo los parámetros establecidos el programa del curso. Al final de cada ciclo, cada docente pone su porcentaje correspondiente a su $50 \%$ y la suma de ambos da la nota final del curso que debe ser igual o mayor a 7.

La propuesta para los cursos del I Nivel es la evaluación integral por medio de un proyecto, donde se evalúen las habilidades que pedagógicamente se han venido enseñando paralelamente y que van de la mano con los objetivos del curso. En otras palabras, un proyecto que integre todas las áreas estudiadas y cuyo enfoque sea dirigido al fin último del idioma: su funcionabilidad práctica y holística.

Contrario al primer nivel de la carrera, los estudiantes se enfrentan a una realidad muy distinta en los niveles superiores. Para empezar los estudiantes ya no reciben ningún curso donde se integren las habilidades del idioma; al contrario, las áreas del inglés se dividen por especialidad. Además, en los niveles superiores y hasta la culminación de la carrera, la ramificación de los cursos se vuelve más especializada y puntual. Por naturaleza de los programas de estudios universitarios, se entiende que se deba dar de esta manera. No obstante, es el deseo de esta propuesta unificar los criterios en conjunto de evaluación hasta donde la malla curricular de la carrera lo permita. A continuación se presenta la distribución de los cursos por nivel para luego dar pie a la propuesta de evaluación integral para cada uno, evaluación que se basa en proyectos finales generalmente de investigación.

\section{Nivel}

Primer Ciclo (duración 17 semanas)

Cursos:

Pronunciación Vocales y Aspectos Prosódicos

Gramática Básica

Lengua y Cultura

Composición: Párrafo

\section{Elocución}

\section{Nivel}

Segundo Ciclo (duración 17 semanas)

Cursos:

Expresión Oral: Sociedad y Humanismo

Pronunciación Consonantes

Gramática Intermedia

Composición: Ensayo

Entonación

Lectura

\section{Nivel}

Primer Ciclo (duración 17 semanas) 


\section{Cursos:}

Expresión Oral: Ciencia y Tecnología

Lingüística General I

Gramática Avanzada

Lectura Académica

\section{Nivel}

Segundo Ciclo (duración 17 semanas)

Cursos:

Expresión Oral: Comercio y Economía

Introducción a la Literatura Inglesa

Taller de Expresión Escrita

Fonética y Fonología

Lingüística General II

\section{Nivel}

Primer Ciclo (duración 17 semanas)

Cursos:

Literatura y Cultura de los EEUU

Comunicación Intercultural

Investigación Bibliográfica

Morfología y Sintaxis

\section{Nivel}

Primer Ciclo (duración 17 semanas)

Cursos:

Investigación de Campo en inglés y Producción Educativa

Literatura Victoriana o Poesía, Drama y Tragedia

Diacronía de la Lengua Inglesa

Seminario de Innovación

Expresión Oral Avanzada

(Nota: Es preciso aclarar que en este apartado no se escribieron los cursos pedagógicos, ya que la propuesta de evaluación integral solamente está dirigida en organizar un equipo de trabajo en los cursos impartidos por docentes de la ECLC de la UNA y en mejorar la lengua meta: el inglés).

Observando la distribución de los cursos de lengua por niveles, se puede apreciar una segmentación y por ende no existe una interrelación evaluativa de habilidades de la lengua, tales como las del primer año. En consecuencia y naturalmente, los estudiantes comienzan a sentir inclinación por unas áreas más que por otras, según sea su afinidad, dejando de lado la apreciación del idioma inglés como un todo.

En discusiones académicas en la ELCL se ha puntualizado lo anterior como una debilidad porque (dejando de lado las destrezas de cada estudiante), la tendencia es estudiar para la aprobación de un curso X y no el aprendizaje integral del idioma. En algunos esfuerzos de integración, los académicos de la ELCL de la UNA han acordado corregir los errores que los estudiantes presentaran en cualquiera de las habilidades del idioma sin importar el área de especialidad que este tenga. Por ejemplo, en años anteriores, si un profesor de composición observaba que sus estudiantes al hablar cometían muchos errores, pero al escribir no se hacían presentes, éste no tenía rubro específico donde evaluar dichos errores. Podía corregir, pero no descontar puntos por los errores cometidos. Tal acuerdo ha venido a darle la potestad de incluir un pequeño porcentaje del $100 \%$ del curso y así observar el uso del idioma dentro de su curso. Este tipo de acuerdo ha contribuido a la integración del idioma de forma holística; pero todavía existe un vacío importante. Por lo anterior, la propuesta 
de una educación y evaluación integral por medio de proyectos en algunos cursos afines de los diferentes niveles es sin duda una táctica metodológica de la cual los estudiantes podrían verse muy beneficiados; ya que la pueden trabajar a lo largo del semestre, optimizan recursos y tiempo y la retroalimentación que obtendrían de tres o más docentes puede ser muy provechosa.

Un vistazo a la malla curricular del BEI ha dado pie a la propuesta de evaluación integral por medio de un proyecto que se llevará a cabo en un trabajo interdisciplinario por los docentes de nivel. Se propone que los cursos de expresión oral, escritura, gramática y cultura trabajen este tipo de la metodología propuesta en la evaluación integral por proyecto.

Se pretende que los profesores de los cursos supra mencionados estudien la programación de cada uno de los cursos en conjunto, y así obtengan una idea clara de lo que cada docente va a realizar en su clase.

Al poseer este tipo de conocimiento, la puesta de un proyecto en común debe ser creada, establecida y coordinada por los docentes de los cursos tomando como base los contenidos de los cursos de expresión oral, partiendo de ahí con la premisa para establecer el tema a tratar en el proyecto.

Cada docente guiará el proyecto de acuerdo a su especialidad. El tema será el denominador común que debe ser desarrollado a nivel oral y escrito, haciendo uso de las estructuras gramaticales de acuerdo al año de la carrera que se cursa y tomando la cultura como un eje transversal, según sea el caso particular de cada nivel.

Es importante recalcar que la creación del proyecto, debe quedar especificada en el programa del curso, dentro de los apartados metodología y rubros de la evaluación y que posteriormente se debe entregar a los estudiantes una guía de trabajo especificando de manera detallada los pasos del proyecto.

\section{¿Qué tipo de proyecto debe realizarse?}

Hablar de qué proyecto se ajusta mejor a esta metodología de trabajo puede ser muy amplio. Sin embargo, basándose en las características y principios de cómo realizar trabajos de proyectos debe tener dos ejes principales, la investigación y el estudiante como eje actoral. Algunas sugerencias para posibles proyectos de trabajo integral evaluativo pueden ser:

1. Reflexiones sobre textos o libros literarios.

2. Trabajos de Investigación donde se realicen encuestas, observaciones participativas o no, entrevistas.

3. Trabajo de campo a nivel cultural, lingüístico o socio-lingüístico.

4. Análisis de videos, documentales o series.

5. Análisis de registros lingüísticos, regionalismos o expresiones.

6. Análisis del discurso escrito vs el discurso oral.

Los ejemplos de trabajos expuestos en la lista previa son algunos de muchos otros que pueden realizar los discentes, sin embargo el hilo conductor de cada uno está orientado a la indagación del tema y al análisis. En este último punto entran la experticia de los docentes. Establecer la guía de trabajo, los objetivos, los instrumentos a desarrollar en caso que se requieran, los puntos a evaluar dependiendo del curso específico y el resultado deseable para el curso en sí y a nivel individual. La evaluación porcentual es muy importante puesto que el objetivo de los estudiantes en su mayoría es aprobar los cursos. Por tanto, debe definirse claramente y explicarla a los aprendices.

\section{Criterios de evaluación del proyecto}

Siendo el proyecto coordinado por varios colegas, la evaluación porcentual cobra una gran importancia. Esa así como se propone la siguiente distribución porcentual.

Que cada profesor asigne de un 5\% a un $10 \%$ de la nota final para el proyecto en común, así mismo, que cada profesor evalúe el producto utilizando su propia rúbrica la cual debe dar a conocer como parte del programa de curso. Además, cada docente debe de contemplar el proyecto en el programa de curso tanto en la metodología como en la evaluación. La evaluación final se realiza una 
vez concluido el proyecto, determinando si los resultados de las actividades desarrolladas en función de los objetivos propuestos originalmente se lograron. El docente debe otorgar criterios para definir el avance, logros, y alcance que cada aprendiz haya desarrollado durante su transcurso de enseñanza-aprendizaje a nivel individual e integral en los procesos de desarrollo de la lengua meta.

\section{Aspectos a tomar en cuenta para el proyecto}

Para ejemplificar un poco más en detalle sobre la propuesta de un proyecto de una evaluación integral para las diversas áreas del inglés, se debe de incluir las siguientes valoraciones en el desarrollo del mismo:

\section{Área de Gramática}

Los siguientes puntos deben tenerse en cuenta a la hora de aplicar el proyecto de evaluación integral entre los profesionales de experiencia lingüista con respeto a la gramática.

Sintaxis:

El profesor se asegura de que los estudiantes hagan uso de las estructuras estudiadas durante el curso del nivel correspondiente. Al trabajar en una tarea o proyecto final, cada instructor académico tiene que identificar y explicar a los estudiantes como la gramática será usada en el proyecto.

\section{Estructuras:}

El profesor de gramática tiene que comunicar las estructuras que deben ser utilizadas en el proyecto final las cuales están ligadas al curso gramatical que se esté impartiendo en el nivel. Esto con la intención de poner en práctica lo que está siendo aprendido en el curso buscando la unificación de las áreas en la enseñanza de un segundo idioma.

Evaluación:

El instructor de gramática debe guiar a los estudiantes en el desarrollo de ambos: las tareas orales y escritas. En otras palabras; el profesor de gramática tiene un propósito doble; uno de guiar al estudiante a utilizar las estructuras gramaticales adecuadas para el nivel, y el otro a colaborar en la integración del proyecto que refleja el lenguaje en su conjunto por el cual todas las áreas de la lingüística estarán así relacionadas.

\section{Áreas de Producción Oral}

A continuación se presentan los puntos que deben tenerse en cuenta a la hora de aplicar el proyecto de evaluación integral por los profesionales de experiencia lingüística con respecto al área de la producción oral.

Estructura de la presentación oral:
a. El estudiante necesita un captador de atención
b. El estudiante debe presentar una introducción que contenga una tesis
c. Cuerpo (defensa de ideas)
d. Resumen
e. Conclusión

Coherencia y Cohesión:

A fin de establecer la coherencia y la cohesión, el estudiante debe introducir el tema con claridad y también referirse a los puntos en detalle con la evidencia y justificación de la investigación. Todo lo que se menciona debe estar vinculado correctamente. La interrelación de todo el proceso tiene que tener una lógica desde el inicio hasta el producto final. El estudiante necesita constante retroalimentación durante todo el desarrollo del proyecto. Tiene una vital relevancia la utilización de 
uso de vocabulario, estructuras y jerga que delimita los contenidos de los cursos partiendo de lo esperado en el nivel.

Fluidez:

Durante la presentación misma, el exponente tiene que tener un monitoreo constante de su fluidez. Por eso, es de suma importancia que se exponga de temas que tenga conocimiento y con el cual el exponente se siente cómodo. Muchas veces los estudiantes eligen temas muy difíciles y terminan por memorizar su presentación. Cuando se hace de esa manera, se corta la precisión lingüística y se afecta la fluidez notablemente. Lamentablemente si el expositor olvidase una palabra, esto podría resultar en olvidar de toda la presentación. La evaluación integral es un elemento esencial aquí, puesto que al fin del ciclo cuando deba presentarse la exposición, el estudiante se va a sentir muy cómodo pues manejará el tema y dominará su producción oral dándole a esta naturalidad y dinamismo.

\section{Conocimiento del Tema:}

El estudiante tiene que demostrar su conocimiento del tema, y esto da pie a que el proyecto (especialmente si es investigativo) debe empezarse desde las primeras semanas del ciclo. Al iniciar el ciclo, los docentes deben de dar a los pupilos las guías de trabajo y las fechas de programación de entrega de adelantos del trabajo. El proceso requiere de mucha comunicación cruzada entre todas las partes involucradas. Primero docente-docente, después docente-alumno, luego alumno-docente y por último para la nota final docente-docente. Hacerlo de esta forma reafirma el conocimiento que va a presentar el estudiante, ya que, los docentes darán los insumos necesarios para que el tema esté justificado, validado y con buena fuente bibliográfica.

\section{Pronunciación:}

La pronunciación debe ser precisa durante toda la presentación. El estudiante debe de mostrar un buen ritmo y entonación, que coincida con el nivel donde se esté desarrollando el proyecto. Es de suma importancia que los estudiantes mantengan un monitorio de su producción lingüística. Adquirir conciencia de sus errores y debilidades es una arma poderosa para el mejoramiento de la adquisición de una segunda lengua donde aspectos como la entonación, ritmo, fluidez, entre otros se vuelven tan naturales que, en la mayoría de los casos, se puede crear una introspección lingüística del idioma meta.

\section{Citas Bibliográficas:}

Es de vital importancia que los estudiantes reconozcan sus fuentes cuando vayan a exponer su trabajo. El número de fuentes dependerá del nivel donde se lleve a cabo el proyecto, pero el reconocimiento de las fuentes da validez al trabajo y lo vuelve fiable. Incluir las citas bibliográficas en sus trabajos es imperativo pues que ellas contribuyen a los trabajos que se presentan, además que no hacerlo se convierte en plagio pues se obvian los derechos de autor.

Motivación:

Los estudiantes deben ser conscientes de que tienen que ser entusiastas mientras que están presentando su discurso. Los gestos, movimientos corporales y el contacto visual son muy importantes para mantener la atención de la audiencia. La preparación con anticipación ayuda mucho a que los estudiantes tengan mayor seguridad para llevar a cabo una presentación bastante autentica.

\section{Área de Escritura}

Los siguientes puntos deben tenerse en cuenta a la hora de aplicar el proyecto final de evaluación integral entre los profesionales de experiencia lingüística con respecto a la parte de producción escrita. 
Elaboración de un Párrafo, una Composición o un Ensayo:

El profesor de escritura debe basar la parte de su proyecto sobre el tema sugerido por el área del curso de expresión oral y asignar la tarea según el curso de composición que éste este enseñando según el nivel.

Procedimiento:

Dependiendo del nivel, el profesor de escritura debe guiar a los estudiantes en la elaboración de un documento escrito en el que evaluará las estructuras gramaticales en estudio, previamente establecidas por el currículo del curso de gramática.

Requisitos del Proyecto:

El profesor de escritura debe brindar una guía con los requerimientos para que los estudiantes realicen la tarea de escritura y el grado de complejidad de la investigación que se llevará a cabo. Todo esto debe ir ligado a los objetivos de los cursos y demandas lingüísticas que presenta cada uno.

\section{Evaluación:}

El profesor de escritura debe dar a conocer a los alumnos cómo se evaluará el proyecto. Esto debe llevarse a cabo por medio de una rúbrica que incluya los objetivos del curso, las estructuras gramaticales y el uso del lenguaje. Además, la rúbrica debe de indicar porcentajes para la coherencia, cohesión ortografía, vocabulario; valores que son parte de la revisión de cualquier trabajo de escritura literaria.

Los aspectos anteriormente explicados son la base primordial para establecer un equipo de trabajo de los especialistas del área de inglés del Bachillerato de la Enseñanza del Inglés de la ELCL en la UNA. La coordinación de todas las partes involucradas es de suma importancia para poder llevar a cabo una evaluación integral a lo largo de la carrera y progresar en la complejidad de los proyectos desde el Nivel I hasta el Nivel IV. Esta metodología de trabajo escriba su objetivo en lograr hacer conciencia en el aprendiz de un segundo idioma (en este caso el inglés) que para una mayor y mejor internalización del idioma, se debe contextualizar este, y esto sirva de rampa no sólo para su crecimiento personal, sino que ayudaría grandemente a su desarrollo profesional. Impulsar una propuesta como esta en la enseñanza del inglés en una institución costarricense podría contribuir muy efectivamente a nivel nacional. El resultado de cambios como este puede generar estrategias metodológicas que cambian los paradigmas establecidos por años relacionado con las enseñanzas de segundas lenguas. Al final, la propuesta no solo busca implementar una evaluación integral; sino, busca (quizás) aliviar uno de los vacíos existentes en la enseñanza de los idiomas en el país. Una enseñanza que se concentra en metodologías rígidas unilaterales (profesor- conocedor alumnoreceptor) cuyo proceso enseñanza-aprendizaje tiende a tener resultados menores o a más largo plazo. Se concluye que una propuesta de este tipo puede traer beneficios muy positivos a nivel institucional, personal y socio-educativo.

\section{Conclusiones}

La evaluación integral no es una metodología que ha salido de la nada, sino que es el resultado de muchos intentos por mejorar los sistemas educativos. La inclusión de ejes transversales, la implementación de programas establecidos por tópicos, la incorporación de aspectos socio-culturales, cultura e interpretaciones semióticas son algunos ejemplos reales que los pedagogos y académicos han utilizado para crear una educación más integral en general. Todo esto ha servido para crear diferentes modelos de enseñanza; por ende, varias formas de evaluar los conceptos que se van adquiriendo. Por tanto, se puede concluir que una evaluación integral en la enseñanza de un segundo idioma es:

1- Una metodología evaluativa que contextualiza los conceptos y funciones que se enseñan en un programa de estudios.

2- Un sistema que involucra el contexto sociocultural de los elementos del lenguaje y que enfrenta al aprendiz con su realidad cultural. 
3- Un sistema que esta intrínsecamente ligado al marco cultural-axiológico cuyo flujo de información es cíclico: aprendiz-profesor-aprendizaje repitiendo el ciclo una y otra vez.

4- Un sistema que cambia el estilo organizativo institucional entrecruzando elementos de evaluación organizados por los colaboradores académicos y rompiendo los paradigmas tradicionales.

5- Un sistema cuyo modelo metodológico y pedagógico se basa en el aprender haciendo por medio de proyectos investigativos (en general) y su desarrollo involucra la flexibilidad del modelo de enseñanza-aprendizaje de la colaboración.

6- Un sistema que conduce a forjar nuevos indicadores educativos que reflejen tanto cualitativa como cuantitativamente esas nuevas posibilidades del aprender. 


\section{REFERENCIAS}

Asamblea Legislativa de Costa Rica. (1973). Aprobación de la Ley de la Creación de la Universidad Nacional de Costa Rica. Departamento de Servicios Complementarios, República de Costa Rica.

Contreras, O. L. (2012). Stephen D. Krashen: Sus Aportes a la Educación Bilingüe. Revista Rastros Rostros, 14(27), 123-124.

Goodman, K. (2011). El Lenguaje Integral: Un Camino Fácil para el Desarrollo del Lenguaje. Revista Lectura y Vida, 2.

Howard, G. y Walters, J. (1986). Multiple Intelligences. Nueva York, Estados Unidos: Longman.

Krashen, S. (1988). Second Language Acquisition and Second Language Learning. Reino Unido: Prentice Hall International.

- (1985). The Input hypothesis: Issues and Implications. Nueva York, Estados Unidos: Longman.

Ministerio de Educación Nacional. (2006) Estándares Básicas de Competencias en Lenguaje, Matemáticas, Ciencias y Ciudadanas. Bogotá, Colombia: Ministerio de Educación Nacional.

Orengo, J. (2011). La Educación Integral. Revista Digital de Profesores. Educ. 106, Universidad Metropolitana (UMET). Puerto Rico.

Seibold, J. (2000). La Calidad Integral en Educación: Reflexiones sobre un Nuevo Concepto de Calidad Educativa que Integra Valores y Equidad Educativa. Revista Iberoamericana de Educación, 23.

Ubal, M. (2011). Hacia una Pedagogía de la Educación Integral. Revista Boletim Técnico do Senac: a R. Educ. Prof., 37(3).

Universidad Nacional de Costa Rica. (2015). Programas de Estudios del Bachillerato de la Enseñanza del Inglés. Heredia, Costa Rica: Escuela de Literatura y Ciencias del lenguaje.

WittgensteinI, L. (1999). Tractatus Logico-Philosophicus. Madrid, España: Alianza.

Yus, R. (1998). Temas transversales: hacia una nueva escuela. Barcelona, España: Graó.

\section{SOBRE LOS AUTORES}

Natin Gerardo Guzmán Arce: Bachiller de la Universidad de Costa Rica, Licenciado en Educación con Énfasis en Inglés de Universidad Estatal a Distancia de Costa Rica, Maestría en Inglés como Segundo Idioma en Universidad Latina Costa Rica (Laudarte International Universities). El nivel de trabajo comprende todos niveles educativos en el sistema costarricense: primaria, segundaria y universitario. He trabajo desarrollando programas educativos para el Ministerio de Educación Pública Costarricense, elaboración de cursos de inglés con Propósitos Específicos para banca, taxistas, turismo, médicos, abogados, etc. Elaboración de guías académicas para los cursos de inglés impartidos en la Universidad Nacional en gramática, expresión oral y escrita y comprensión auditiva. Temas de interés: cultura y sociedad, la sociolingüística, el desarrollo humano, idioma y cultura, técnicas de enseñanza de aprendizaje.

Mayra Loaiza Berrocal: Bachiller en Inglés de la Universidad Internacional de las Américas, Licenciada en Lingüística Aplicada a la Enseñanza del Inglés como Segundo Idioma de la Universidad Nacional de Costa Rica y Máster en Segundas Lenguas y Culturas de la Universidad Nacional de Costa Rica. Su experiencia laboral ha sido en la formación de educadores del idioma inglés en la Universidad Nacional de Costa Rica, coordinadora de los Cursos libres de la Escuela de Literatura y Ciencias del Lenguaje en la UNA, coordinadora de los programas de estudios de la Escuela de Literatura y Ciencias del Lenguaje. Temas de Interés: cultura y su enseñanza, sociedad y humanismo, sociolingüística. 